\title{
Implementation of e-education in Africa via space digital video broadcasting system
}

\author{
Dimov Stojce Ilcev \\ Space Science Centre (SSC), Durban University of Technogy (DUT), Durban, South Africa
}

\begin{tabular}{|c|c|}
\hline Article Info & ABSTRACT \\
\hline $\begin{array}{l}\text { Keywords: } \\
\text { DVB-RCS } \\
\text { DVB-S } \\
\text { DVB-S2 } \\
\text { Ground segment } \\
\text { Hub } \\
\text { Space segment } \\
\text { VDVoIP } \\
\text { VoIP } \\
\text { VSAT }\end{array}$ & $\begin{array}{l}\text { This paper introduces an advanced e-education provision via space systems } \\
\text { for Africa or any other regions in remote dispersed communities, such as rural, } \\
\text { mining, agriculture, surveying, construction, tourism, military and etc. } \\
\text { Based on the specific needs and requirements e-education implies significant } \\
\text { broadband applications, interconnectivity, and timely and quality-assured } \\
\text { content delivery of service. The e-education solutions of distance learning } \\
\text { and training for remote and rural areas, which are beyond range of terrestrial } \\
\text { and short distance wireless cellular facilities, cannot provide broadband access } \\
\text { without space-enabled communication solutions, such as satellite } \\
\text { constellations and stratospheric platform systems (SPS) or high altitude } \\
\text { platforms (HAP). This paper also discusses the integration challenges that are } \\
\text { presented by combining space solutions for implementation e-education and } \\
\text { learning in rural and mobile environments. Configuration of in-house } \\
\text { development of all segments, installation of the scale-down digital video } \\
\text { broadcasting-return channel via satellite (DVB-RCS) hub (gateway), ground } \\
\text { network and very small aperture terminal (VSAT), known as fixed interactive } \\
\text { terminals (FIT), for e-education, distance learning and staff training initiative } \\
\text { in Africa are described. }\end{array}$ \\
\hline & This is an open access article under the $\underline{C C B Y-S A}$ license. \\
\hline \multicolumn{2}{|l|}{ Corresponding Author: } \\
\hline \multicolumn{2}{|c|}{$\begin{array}{l}\text { Dimov Stojce Ilcev, } \\
\text { Space Science Centre (SSC), } \\
\text { Durban University of Technogy (DUT), } \\
\text { 41/43 M L Sultan Rd, Greyville, Durban, 4001, South Africa. } \\
\text { Email: ilcev@ dut.ac.za }\end{array}$} \\
\hline
\end{tabular}

\section{INTRODUCTION}

In the past are designed many proposals and projects deploying ICT and software support to provide e-education in remote environments, but these solutions couldn't work at all without implementing two-way space connectivity via geostationary earth orbit (GEO), medium earth orbit (MEO), low earth orbit (LEO), SPS or unmanned aerial vehicles (UAV). Usually the best satellite solutions for e-education are GEO satellite constellations deploying DVB-RCS standards and SPS solutions with new proposed digital video broadcastingreturn channel via platforms (DVB-RCP) technique and technologies. In addition, it will be necessary to examine a set of services and infrastructures that will realize e-education and distance learning systems for rural schools, trainings facilities for corporate organizations and to assess the targeted users' interest in such specific applications.

The growing demand for satellite or platform communications bandwidth over all wireless media has motivated the introduction of digital satellite communications solutions that will provide bi-directional services at greater than $300 \mathrm{MB} / \mathrm{s}$ known as DVB-RCS or DVB-RCP. Current DVB applications may demand rapid 
e-education installations at fixed locations of urban, suburban and rural environments, as well as mobile platforms, such as buses or vehicles for e-learning [1, 2].

In Figure 1 are illustrated all possible solutions to relay e-education links from urban to rural areas via GEO, MEO, LEO, SPS airship stations, UAV aircraft platforms. In fact, more than a basic introduction, the DVB project presents the specific approaches to design and select an appropriate broadband satellite constellation using $\mathrm{C}, \mathrm{Ku}$ and $\mathrm{Ka}$-bands, to configure ground elements, evaluate sources of antennas technology, radio frequency (RF) electronics equipment, services and solutions. In fact, this new technique can take into consideration all applications to commercial and government users as well as compliance with international radio regulations of the International Telecommunication Union (ITU).

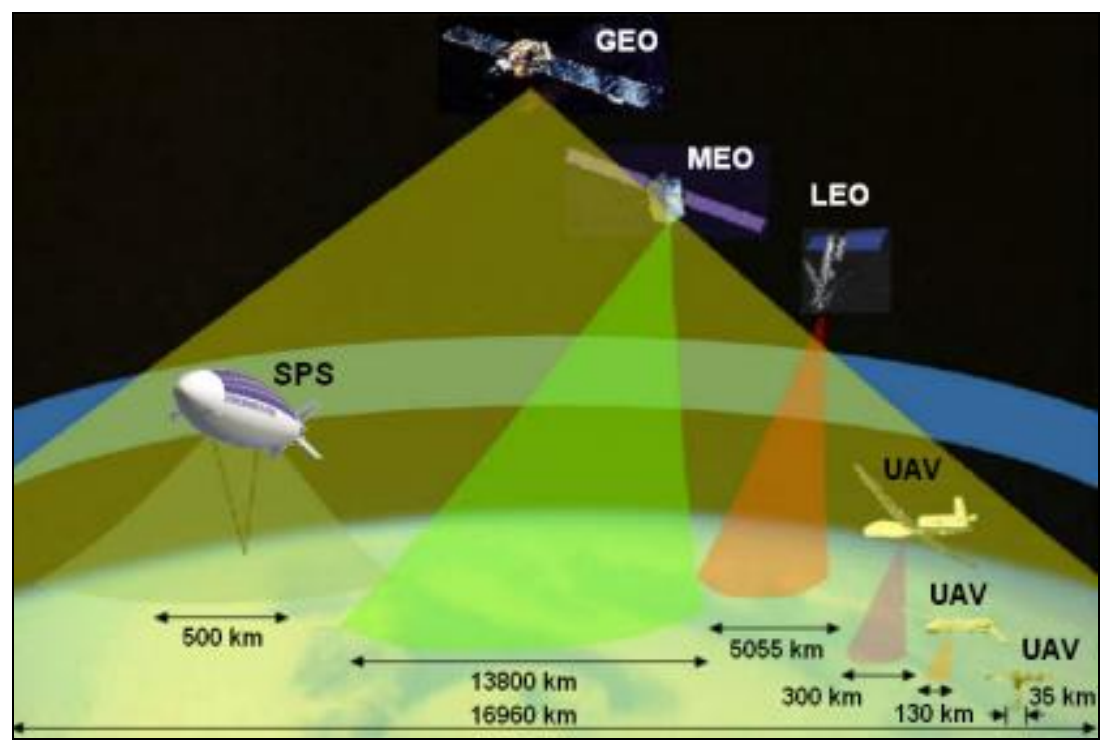

Figure 1. Possible space constellations in function of e-education

\section{E-EDUCATION VIA STRATOSPHERIC PLATFORM SYSTEMS (SPS)}

The SPS technologies will provide implementation of DVB-RCP access via airship for fixed and remote broadband communications connecting urban, suburban, rural and mobile platforms anywhere and in anytime. Thus, the SPS network contains space and ground segment and one airship can cover up to $500 \mathrm{~km}$ in radius The development of the DVB-RCP network for e-education will need design of special airship that will act as geostationary very low orbit satellite at about 20 to $50 \mathrm{~km}$ in stratosphere. Space segment can include one or few airship platforms connected via inter platform links (IPL) using laser or optical communications. Deploying few airships with overlapping coverage will be covered a large land masses over one country. The ground segment consists on one side hub, ground earth station (GES) or gateway interfaced to the terrestrial telecommunication network (TTN), such as internet service providers (ISP), integrated service digital network (ISDN), broadband ISDN (B-ISDN), asynchronous transfer mode (ATM), universal mobile telecommunications system (UMTS), general packet radio services (GPRS), G4 or G5 cellular networks and broadcast e-learning centre (BEC).

On another side are connected users segment of rural schools to the BEC site via one or few airship platforms. Thus, educators in BEC can provide lessons of many subjects in the real time via Videoconference (VC) to the rural classrooms equipped with DVB fixed interactive terminal (FIT) or very small aperture terminals (VSAT). On the roof of rural school will be fixed DVB antenna as an outdoor unit (ODU) and inside of school will be installed DVB router or indoor unit (IDU). Router is able to connect up to 100 PC terminals in local area network (LAN) by the cable lines or via Worldwide Interoperability for Microwave access (WiMAX) and wireless fidelity (Wi-Fi) by the wireless links. The video lessons coming from BEC in rural schools can be viewed via PC or common big VC screen and educator for each subject will be able to send soft copies of books and other learning materials. Pupils in rural schools can watch IPTV (IP television) as well [3-5].

The e-education infrastructure via SPS solution is shown in Figure 2 with space segment consisting SPS stations and ground segment, which includes DVB-RCP hub with antenna system. As stated, the hub infrastructure is connected to BEC offices broadcasting e-learning centre from where all educators teach a

Implementation of e-education in Africa via space digital video broadcasting system (Dimov Stojce Ilcev) 
variety of subjects that need to be delivered via SPS stations to rural classes via videoconference. On the other side, hub is connected via ISP links to urban customers that needs interactive connections with other sites in rural and remote environments.

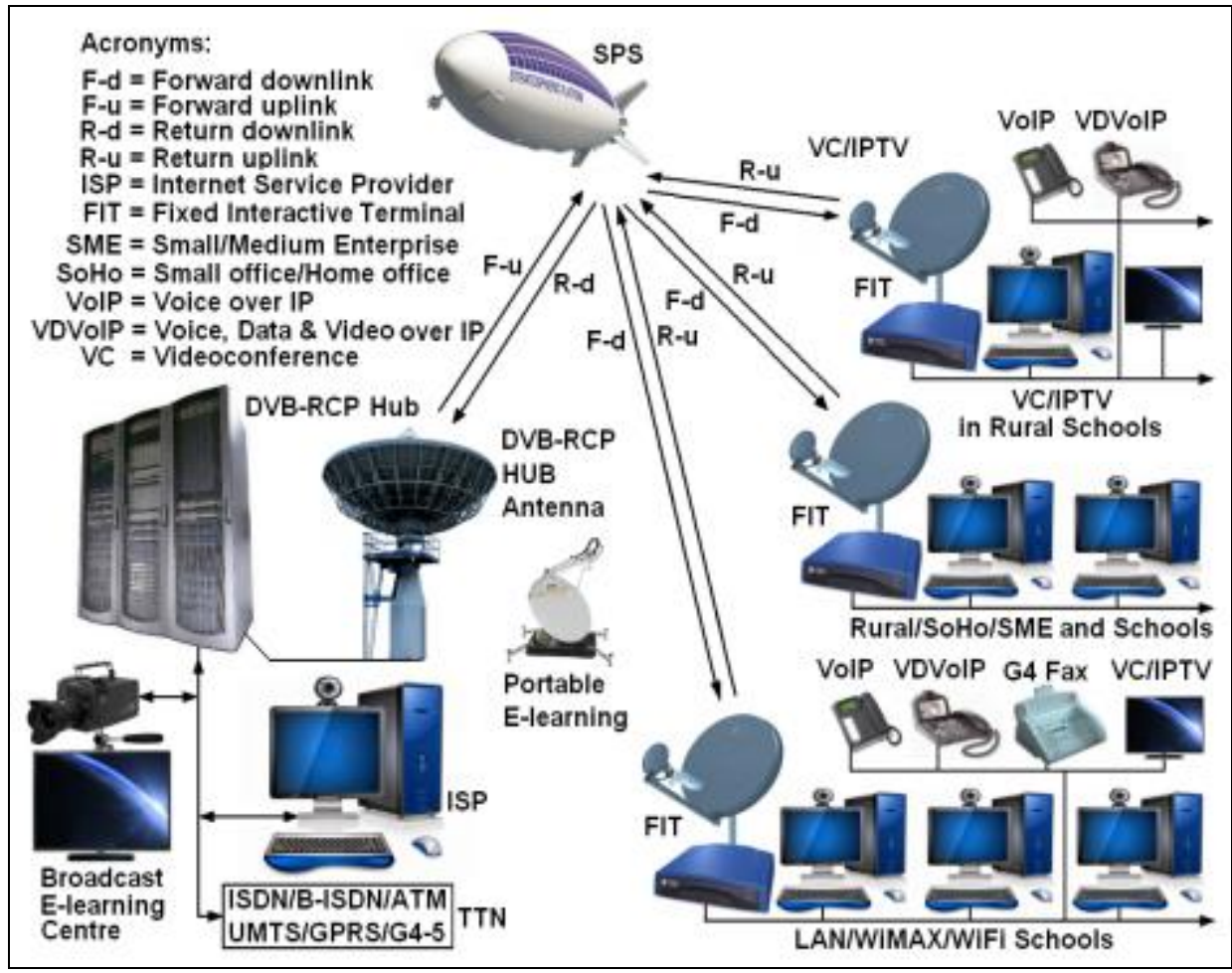

Figure 2. E-education solution via SPS airship

The schools offices, rural households, small offices/home offices (SoHo), small/medium enterprises (SME), rural Internet kiosks and other sites will be connected to internet, VC, IPTV, VoIP telephones, voice, data and video over IP (VDVoIP) units and G4Fax machines. Managers of schools may be in touch their supervisors in Education Department and educators in BEC. In the same time, as alternative can be used portable e-learning VSAT. Therefore, residents in rural areas, households, SoHo and SME customers will be able to deploys own FIT equipment with antennas installed on the roof, to watch IPTV and connect their desktops and laptop to the internet.

From the geometrical point of view, SPS terminals would enable rural communication services that take much advantage of the best features of both terrestrial and satellite multipurpose communications. In addition, however, the system could bring advantages of its own, not available in current systems. The most important advantages of employing SPS are high elevation angles, broad coverage, low propagation delay, low-cost operation, easy and incremental deployment and ability to move around in emergency situation.

The SPS airship is launched using a specified volume of helium separated from the air to maintain its shape. As the platform rises the helium expands and at the proper altitude displaces all of the air within the airship. Once it is in the stratosphere the airship is remotely controlled and moved into determined position. The launch of SPS into position is much simpler than putting a satellite into any orbit. After careful preparation in the hanger space, the airship is launched in 4 Ascent phases through the troposphere and Interface location point in the stratosphere and finally, it shifts to the station-keeping position. The recovery phase goes in the opposite direction, namely, the airship is slowly moved from the station-keeping position towards the interface point and from there descends down to the ground in 4 descent phases $[1,2,5]$.

The SPS airships do not interfere aircrafts flights, because they are located over $10 \mathrm{Km}$, airship itself leverages lighter-than-air (LTA) technology being made of very high strength and lightweight materials. Airship is unmanned and solar powered platform, accompanied by advanced propulsion systems that maintain proper positioning, it is equipped with autonomous navigation, radio controlled command and communications payload stabilization systems. A combination of solar cells, batteries and fuel cells will power the SPS during 
its five-year planned deployment. Thus, it also incorporates telemetry to remotely transmit data (TT\&C) and redundant systems to serve as back-up measures, then features that are designed to provide the airship with a high level of availability, reliability and safety. The SPS is being designed to hold approximately $1,000 \mathrm{~kg}$ of communications payload capable of supplying focused fixed and mobile broadband, narrowband and wireless backbone services to approximately 3 million subscribers.

Although immature airship technology, stabilization and antenna system on the platform are challenging that has to be investigated, the SCP is expected to avoid some inherent limitations belongs to the traditional systems and to provide backbone to cellular network. Those are in the sense of a huge number of base stations required by the terrestrial system, limitation of the minimum cell size on the ground involved in GEO satellite system, and suffer from handover problem faced by LEO/MEO satellite system. With these great advantages, the ITU has allocated the spectrum to SPS (HUP) networks at $2 \mathrm{GHz}$ for $3 \mathrm{G}$ mobile systems, $48 / 47 \mathrm{GHz}$ for the usage worldwide, and $31 / 28 \mathrm{GHz}$ band is allocated for usage in many countries [3, 4].

\section{E-EDUCATION VIA GEO SATELLITE CONSTELLATION}

The first generation of DVB-S standards known as an DVB-RCS, 20 years ago, quickly became one of the key solutions in almost every new advanced satellite communication projects for broadcasting interactive applications, high-speed internet and IPTV. Three years later was designed the second generation of DVB-S2 CCM (constant coding modulation) standard as a more cost effective, efficient, reliable, secure and functional solution. The DVB-S2 CCM is recently upgraded by the most technical and cost effective DVB-S2 ACM (adaptive coded modulation) platform, forward and reverses compatible, which comparison is shown in Figure 3.

The latest DVB-S3 third generation of DVB-S standard was proposed by the Israeli company NovelSat as more efficient successor to the DVB-S2 transmission system. After launching its new DVB-S3 (NS3) modulation technology in April 2011, as more efficient than DVB-S2, start-up NovelSat Company has already established 32 live satellite trials. The system firstly was deployed in a series of VSAT terminals and targeted as a replacement technology for DVB-S2 delivering VDV, Internet and IPTV from the sky to consumer homes and mobiles in remote areas.

Using DVB-S2 does not only cost less, it also allows professional users to do more, while variable coding and modulation (VCM) mode of DVB-S2 transmits several signals on the same carriers, each with his own coding rate and modulation scheme. This mode automatically charges the modulation and forward error correction (FEC) of a link referred as modulation and coding (MODCOD). For instance, the high efficiency of DVB-S2 therefore well needed to squeeze 5 to $8 \mathrm{HDTV}$ channels in $36 \mathrm{MHz}$ satellite communication transponders [6-9].

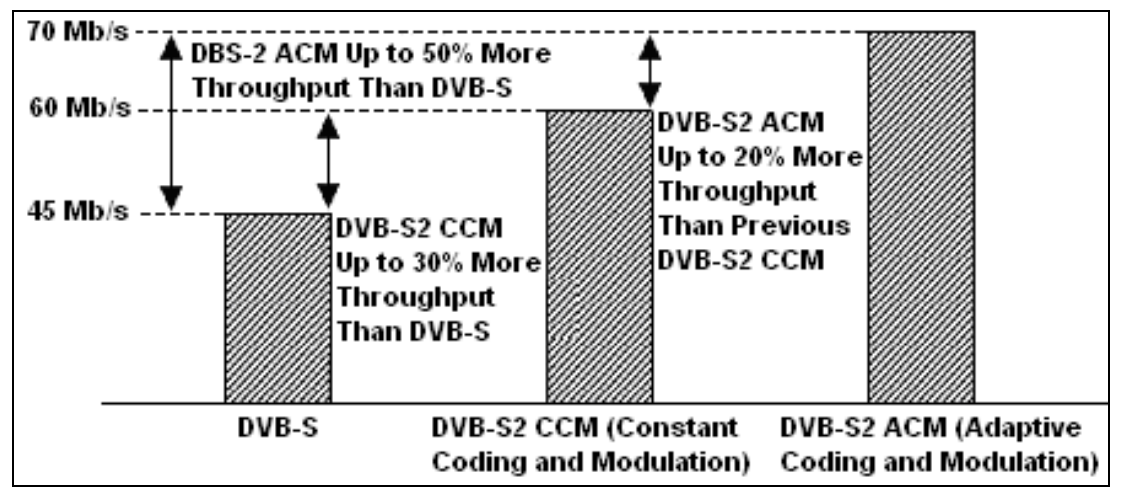

Figure 3. Comparison of DVB-RCS standards

Early results have also shown that using DVB-S2 in VCM mode for IP trunking network could improve the bandwidth efficiency by up to $70 \%$ compared to DVB-S. With VCM can be dynamically selected MODCOD for each IP packet in function of the instantaneous receive conditions at each site, effectively using the gain margin to increase the efficiency by up to $130 \%$ in average. For DVB-RCS systems, this gain will be a decisive factor in the tough competition against terrestrial Internet access services such as ADSL or cable. However, the newest DVB-S2 with CCM (constant coding modulation) and ACM (adaptive coding modulation) are providing $30 \%$ and $50 \%$ mote throughout than first generation DVB-S, respectively, which is shown in Figure 3 [6].

Implementation of e-education in Africa via space digital video broadcasting system (Dimov Stojce Ilcev) 


\subsection{Configuration of DVB-RCS space segment}

For establishment DVB-RCS network in Northern and Southern Africa can be employed existing satellite operators providing the $\mathrm{C}, \mathrm{Ku}$ and $\mathrm{Ka}$-band satellite constellations suitable for DVB-RCS S and S2 scenario, which beam coverages are shown in Figure 4 (left) of Eutelsat and (right) of Arabsat. There are many other DVB satellite operators such as Intelsat, SES-NewSkies, PanAmSat, Telesat, Inmarsat and others providing broadcasting service via GEO satellites.

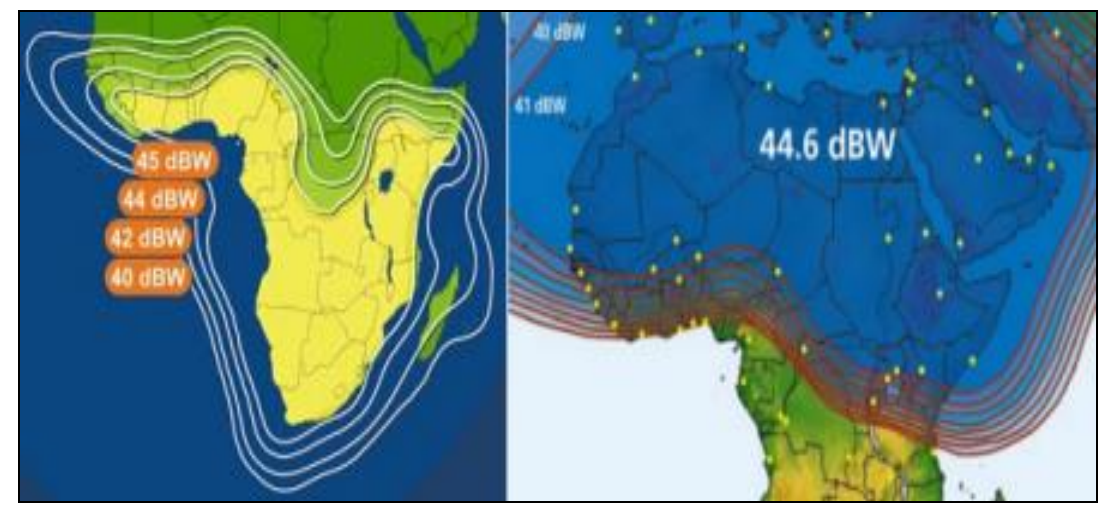

Figure 4. DVB space segment coverages

The British company Inmarsat awarded a contract to the US Boeing in August 2010 to design, build and supply three Inmarsat-5 communication satellites as part of an 752M $£(1.2 \mathrm{~B} \$)$ wireless broadband network known as Global Xpress. The wireless broadband network will operate at Ka-band frequencies ranging between $18 \mathrm{GHz}$ and $31 \mathrm{GHz}$, which however possess more signal quality issues, compared to that of old $\mathrm{Ku}$-band $(12 \mathrm{GHz}-18 \mathrm{GHz})$ frequency range.

The DVB satellite operators provide interactive on-demand regional or global and spot beam coverage for broadband transmissions via GEO satellite constellations. Presently in Africa are operational Egyptian multipurpose spacecraft Nilesat and Nigerian NigComSat. Africa and Middle East have 69 countries, so dividing $1.2 \mathrm{~B} \$$ with 69 will be participation of about $17 \mathrm{M} \$$ per each country in the region to build 3 and 5.5 M \$ for 1 multipurpose GEO spacecraft.

The DVB-RCS space segment may consist minimum three multipurpose GEO satellites consisting communication and navigation transponders. A GEO satellite has a circular orbit in the equatorial plane, with an orbital period equal to the rotation of the Earth of 1 sidereal day, which is achieved with an orbital radius of 66,107 (Equatorial) Earth Radii, or an orbital height of 35,786 km. Otherwise, a satellite in a GEO will appear fixed above the surface of the Earth, and remain in a stationary position relative to the Earth itself. Theoretically, this orbit is with zero inclination and track as a point but in practice, the orbit has small non-zero values for inclination and eccentricity, causing the satellite to trace out a small figure eight in the sky.

The footprint or service area of a GEO satellite covers almost $1 / 3$ of the Earth's surface or $120^{\circ}$ in longitude direction and up to $75^{\circ}-78^{\circ}$ latitude North and South of the Equator but cannot cover the Polar Regions. The near-global coverage can be achieved with a minimum of three GEO satellites in orbit moved apart by $120^{\circ}$, although the best solution is to employ four GEO satellites for better overlapping. This type of orbit is essentially used for commercial communication services for both fixed and mobile applications. The GEO satellite constellation is the best solution for deployment e-education content in any hypothetical country, although also can be used medium earth orbits (MEO) and highly elliptical orbits or their combination with GEO satellites [9-11].

\subsection{Configuration of DVB-RCS ground segment}

The DVB-RCS VSAT satellite network has been designed to minimize the cost of scaling a broadcast, broadband, Internet and multimedia access between hub terminals and hundred or thousands simultaneously logged-on FIT units in rural or remote areas providing e-education via GEO satellite, illustrated in Figure 5. This e-education solution via satellite has almost the same as SPS infrastructure and solutions shown in Figure 2. In addition, the DVB-RCS VSAT network is providing mobile e-learning solutions in special terrain vehicles and in school buses. 


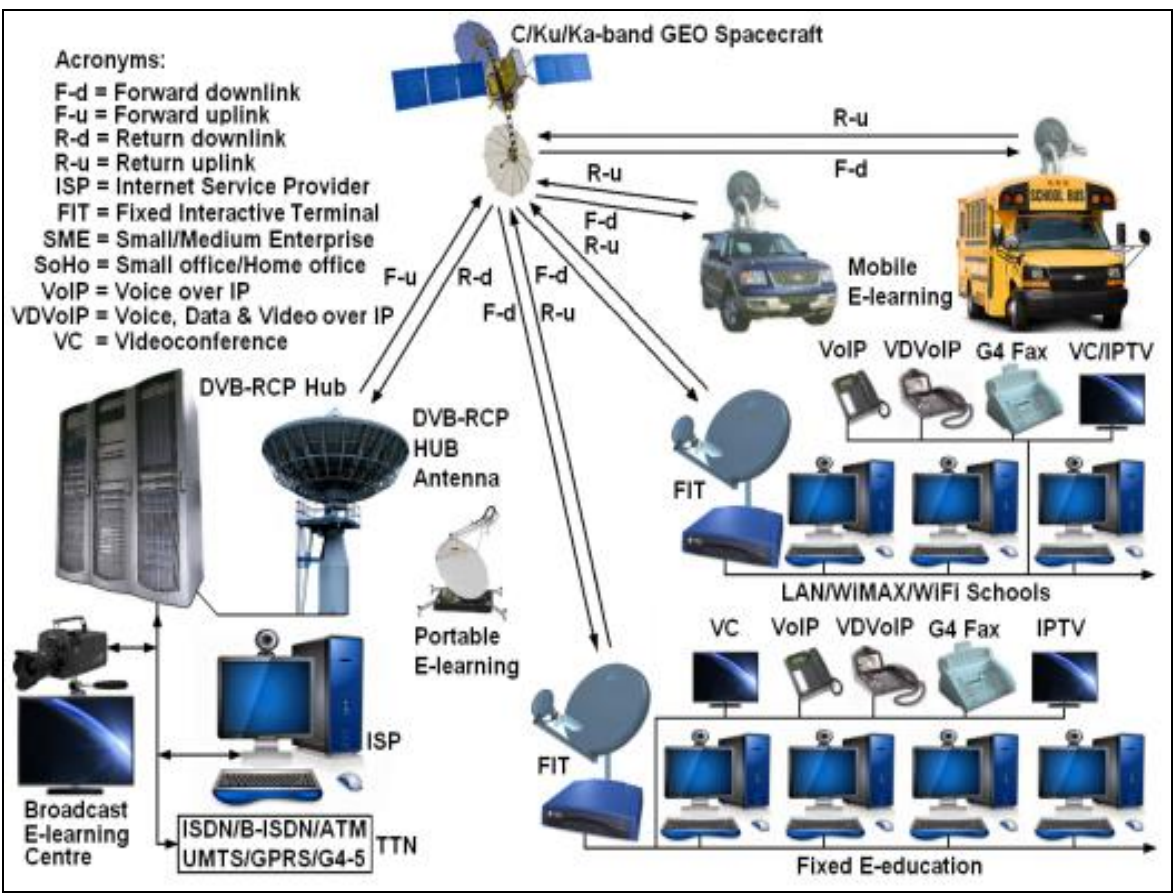

Figure 5. E-education solution via GEO spacecraft

Satellite DVB-RCS via VSAT hub with C (4-8 GHz), Ku (12-18 GHz) or Ka-band (27-40 GHz) antenna system interfaces and extends the terrestrial broadband, video broadcasting, UMTS/GPRS (universal mobile telecommunications system/general packet radio service), asynchronous transfer mode (ATM), TTN, internet service providers (ISP), cellular, private and public networks, virtual private networks (VPT), fiber optical networks (FON) and any other civilian corporate or military networks with corresponding satellite connection to SIT units or remote terminals and enables the following major services:

\section{a. Service provider platforms}

The DVB-RCS hub provides a large fully integrated network management system (NMS) with accounting interfaces network for one or multiple and multipurpose service providers, via one or hybrid satellites, on one or many satellite transponders:

- Regenerate rural and remote communications: internet access and e-mail; telecentres; rural telephony; VoIP, VDVoIP, videoconference over IP (VCoIP); interactive TV and radio two separate way broadcasting; - Electric and distribution networks; community centers; back up to terrestrial networks; GSM backhaul, transport control stations (Tolls); enterprise services to remote offices; VPN with acceleration, village Wi-Fi services to entire communities; SCADA or machine to machine (M2M) monitoring and control, etc.

- Remote teleservice e-medicine and e-education: VCoIP; audio, data and video (ADV), VDV and image transfer; IPTV, VoIP, VDVoIP, interactive distance learning; emergency management platform, remote contribution for live events, distance health diagnostics and medicine, etc [12-14].

b. Enterprises and private networks

The DVB-RCS mini hub standard provides small flexible network with lower cost for up to 20 sites for wide geographic coverage: asymmetric digital subscriber line (ADSL) anywhere and anytime; internet access and e-mail; VDVoIP, VCoIP, VoIP and fax transmissions; consumer/SoHo (small office home office) and SME (small and medium enterprises) LAN, corporate LAN/WAN, intranet/extranet/VPN, data broadcasting and multicasting, banking, digital signage, business TV; enterprise resource planning (ERP) platforms; in-house e-training and education; file transfer protocol (FTP) and hyper-text transfer protocol (HTTP). The FTP scheme is service for moving and copying an electronic file of any type from one computer to another over the internet, for both, downloads and uploads.

c. Broadcasting and content distribution

The DVB-RCS hub standard also enables the following broadcasting services: An overlay to existing broadcast networks; Fast and secure distribution of digital content anywhere, ADV content origination from both fixed and mobile or transportable sites; micro satellite news gathering (SNG) over IP (SNGoIP); software distribution to remote site servers, film distribution to movie theaters; secure intranet VDV communications;

Implementation of e-education in Africa via space digital video broadcasting system (Dimov Stojce Ilcev) 
pay TV services; near video on demand; IPTV delivery and interactive TV and enhanced TV; different onscreen games, program guides and so on.

d. Satellite news gathering (SNG)

The DVB-RCS standard provides SNGoIP as one of the modern tools becoming available to the Broadcasting content and industry. SNG can reduce the cost of gathering content and bandwidth from the field. For instance traditional analog SNG platform cost 100,000 US\$, IP mesh platform costs about 50,000 US\$ and the price of DVB-RCS platform is 30,000 US\$. In fact, it provides the following services: corporate data and disaster recovery; video streaming; multicasting integrated DVD; web casting; push/pull data delivery; in-field military data communications; accident and emergency response; video monitoring of remote sites and so on. e. Satellite emergency and security management

The DVB-RCS standards can provide remote broadband satellite VDV and internet backbone communications for the following homeland security system (HSS) applications: national emergency management solutions; firefighting actions; police operations; search and rescue (SAR) and on-scene communications at sea and on the ground; drug enforcement; disaster management; anti-terrorism; border security; environmental monitoring; interim gateways pending permanent installation; fire training; telemedicine and education (mining, fisheries, oil, exploration, etc); government and military utilities (natural resources management, defense, military, national security).

f. Defense information management

The special DVB-RCS network is unique qualified to supply unparalleled satellite solutions for military tactical and defense applications. This advanced satellite broadband network significantly enhances the performance of any existing military service and enable a host of new applications, with bi-directional transmissions in excess of a T1 (1.544 MH/s) speed line.

The DVB-RCS network enables rapidly deployable fixed and mobile terminals with latest in content-driven applications for the benefit of today's tactical war fighter. For instance, one DVB-RCS hub is able to cover all applications for navy, ground and air forces on the soil of South Africa including entire African Continent and to connect up to 200,000 fixed and mobile terminals with Central and other Command posts, for the following services:

- $\quad$ Rebuilding efforts

- Secure (encrypted) communication

- Logistics management

- Secure (encrypted) communication

- Troop communications

- Logistics management

- E-training and education

Otherwise, the DVB-RCS standard is designed by the European Telecommunication Standard Institute (ETSI) as DVB Project for two-way satellite broadband very small aperture terminal (VSAT) systems. The low cost VSAT equipment can provide highly dynamic, demand-assigned transmission capacity for a broad range of fixed and mobile users. The main features of DVB-RCS2 that this standard provide users are VDV satellite communications and broadband Internet connection, without a need for any local terrestrial infrastructure. The DVB-RCS ground and user segments can be connected via GEO spacecraft and hub or Gateway terminals to the terrestrial internet network (TIN), which architecture is shown in Figure 5. Therefore, GEO satellite is providing foothprint of DVB-RCS spot $\mathrm{C}, \mathrm{Ka}$ and $\mathrm{Ku}$-bands, which includes conections of local fixed (DVB-RCS2+F) networks to the DVB-RCS hub terminals [9, 14-16].

\section{CONFIGURATION OF DVB-RCH HUB TERMINAL}

The hub or gateway terminals with antenna support existing DVB-RCS VSAT compliant forward link system (FLS), but optionally can be provided full FLS, which is illustrated and central component in Figure 5. In fact, the DVB-RCS hubs are turnkey cost effective systems that can be installed in days to enable a wide range of government, corporate and private public network topologies with satellite interactive terminals including rural and learning applications, which two versions are shown in Figure 6(a): ViaSat (left) and Hughes (right). Depending of type, but the biggest hub terminals are able to support up to 80,000 VSAT or router terminals in urban, rural and remote environments, which two versions are shown in Figure 6(a): ViaSat (left) and Hughes (right), and in Figure 6(b) is shown patriot DVB-RCS antenna of Cobham company. 


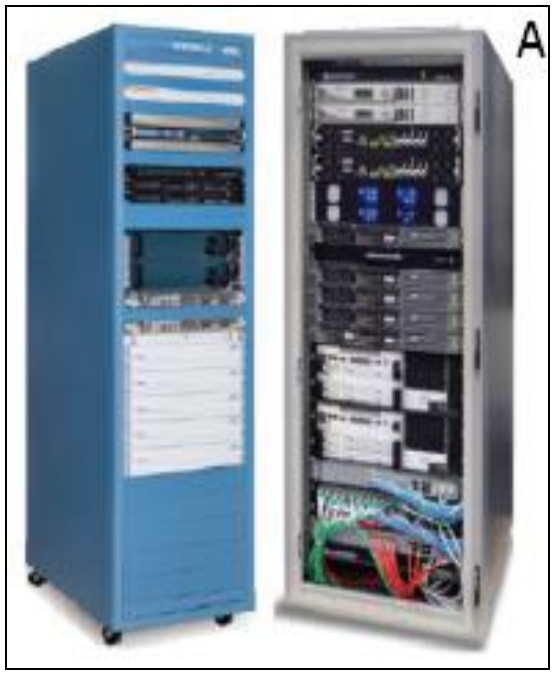

(a)

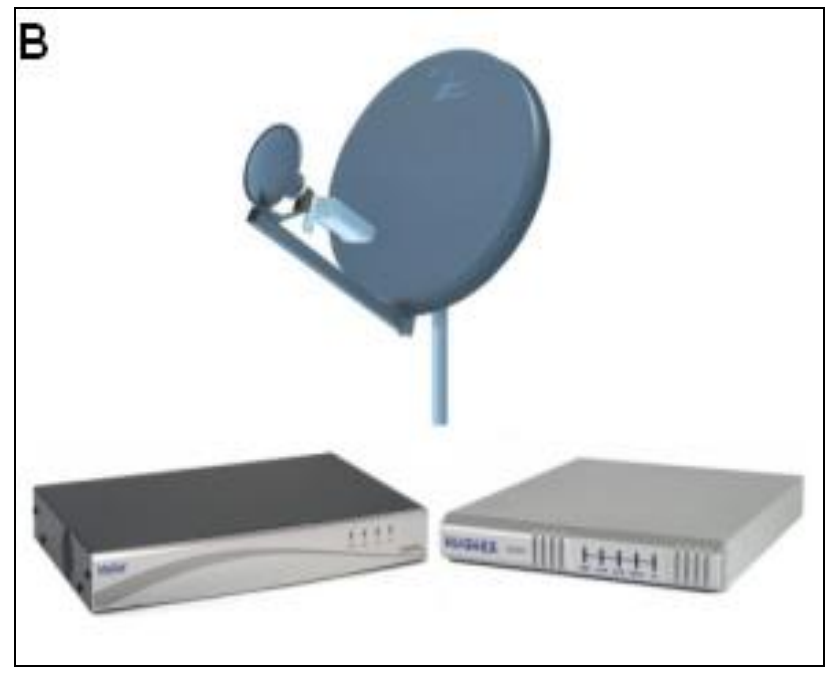

(b)

Figure 6. DVB-RCS ground segment, (a) ViaSat (left) and hughes (right), (b) Patriot DVB-RCS antenna of Cobham company

The DVB-RCS hub main operator can interface FIT terminals to the telecommunication service provider or TTN and manage all the operational aspects of the system. It can be delivered in a number of different configurations to suit customer precise applications. The customer has choice of $\mathrm{C}$, $\mathrm{Ku}$ or $\mathrm{KA}$ antennas and FIT or VSAT RF equipment, what is depending on available satellite transponder RF band. The hub can handles 1 to 10 forward link transmitters and from one to several hundred return link receivers of maximum 80,000 terminals. Its architecture is also designed to accommodate upgrades and expansions of DVB network, and to optimize stability in operation. The main components of the hub terminal illustrated in Figure 5 are as follows:

a. Network control centre (NCC)-The NCC ground configuration assures traffic management, system supervision and protocol handling, and offers sophisticated bandwidth management of the return link through four different bandwidth allocation algorithms based on the DVB-RCS standard. The network management subsystem is the interface between the operator and the hub, using a customer friendly webinterface. Call and management data is stored in system memory and made available towards other operational tools through standard interfaces, e.g. for billing purposes.

b. Forward link subsystem (FLS)-This link consists typically (but not necessarily) of an IP-encapsulator, providing the encapsulation of IP transfer of data packets into MPEG frames, an MPEG multiplexer and a modulator according to the DVB-S transmitting specification. The hub can be delivered with manufacturers providing the new or integrated towards an existing FLS.

c. Return link subsystem (RLS)-This link is the powerful bank of radio receivers, which collect the turbo-coded MF-TDMA bursts transmitted by all the terminals in the network. Each one of RLS receivers can be individually configured to operate at any frequency, bit-rate or coding rate. In this way the hub offers unrivalled flexibility for the operator to maximize the use of air-interface resources. The modular design of the RLS enables the system to scale from small to very large networks.

d. Reference and synchronization subsystem (RSS)-This special equipment delivers the synchronization and timing information in the hub (gateway) terminal for synchronization of the entire satellite network $[9,17-21]$.

\section{CONGFIGURATION OF FIXED INTERACTIVE TERMINAL (FIT)}

The FIT OR remote devices are VSAT interactive (two-way) satellite terminal composed by transceiver (receiver and transmitter), which consists of two main units, the indoor unit (IDU) and the outdoor unit (ODU).

a. Satellite DVB-RCS outdoors unit (ODU) or transceiver antenna

The ODU is transceiver antenna unit shown in Figure 5b (Above), which can be installed on the roof or mast and may provide to indoor unit (IDU) the following features:

- Full DVB-RCS compliance and very easy to install

Implementation of e-education in Africa via space digital video broadcasting system (Dimov Stojce Ilcev) 
- $\quad$ Range of C, Ku or ka-band antenna sizes possible for optimizing system throughput from $0.75-2.4 \mathrm{~m}$

- Standard ODU/IDU interfaces ensure full compatibility with any DVB-RCS or VSAT modem

- Two-way enhanced digital satellite equipment control (DiSeqC) capability, which interfaces between IDU and transmitter supports automatic line-up function at installation.

b. Satellite DVB-RCS indoor unit (IDU) or satellite transceiver

The IDU terminals are the satellite devices designed to connect remote end-user PC LAN and IPTV to a DVB-RCS satellite network, and can be installed in office or with some modification on onboard mobiles. It provides two-way Multimedia IP communications via adequate satellite at $\mathrm{C}, \mathrm{Ku}$ or K-band RF, which two models are shown in Figure 6b (below), ViaSat (left) and Hughes (right).

The IDU terminal can be supplied as desktop with a variety range of data IP throughputs from 4 to 15 $\mathrm{Mb} / \mathrm{s}$. It can serve for government, corporations, institutions, private companies, home offices and e-education offering an open-interface for high-capacity satellite broadband access that bypasses the "last mile" bottleneck associated with terrestrial infrastructure. This system offers satellite broadcast, broadband and multimedia access to core IP ground networks using standard technologies such as DVB-S (DVB-Satellite), DVB-RCS, IP interfacing DVB-T (DVB-Terrestrial) and MPEG/DVB-S or DVB-S2 with user terminals via corresponding $\mathrm{C}, \mathrm{Ku}$ or Ka-band satellite transponder. The DVB-RCS IDU terminals are capable to provide RLSS service up to 100 PC in LAN customer sets simultaneously with Ethernet interface or, let's say PC and IPTV fixed units.

The satellite antenna (ADU) usually can be fixed on the roof and can provide a data rate that reaches speeds of $4 \mathrm{Mb} / \mathrm{s}$ upload and $36 \mathrm{Mb} / \mathrm{s}$ download. Therefore it provides voice, data and video (VDV) connectivity with the DVB-RCS platform or audio/video transmissions. With a simple push of a button, the fixed satellite antenna will be connected onto predetermined satellites, transmitting or receiving the content in less than five minutes of deployment $[9,14,20]$.

\section{E-EDUCATION AND DISTANCE LEARNING OVER DVB-RCS STANDARS}

Improvements in satellite communications technology during the past several years have been making it easier and less expensive for population at multiple locations to be educated, informed and to work and study collaboratively. Interactive DVB-RCS networks make it possible for people to receive better education, job training regardless of their office location, learn from teachers in distant cities and provide medical care even if they cannot physically be with a patient. Even as cellular and terrestrial telecommunications networks become more widely available in urban areas, satellites remain the technology of choice for e-education and distance learning kinds of services, known collectively everywhere as distance classes.

The advent of new highly efficient digital telecommunications techniques, coupled with the rapid build out of fiber-optic and other ground facilities even in less-populous areas, has spurred some users to re-examine their technological requirements and pass via satellite systems whenever possible. And the spread of high-speed Internet connections has allowed people to use personal computers and laptops to enjoy many of the benefits afforded by traditional distance learning via satellite techniques in urban areas. In remote and rural environments is not possible at all to get this facilities just using ICT or IP via satellite, personal computer and some software. Therefore, the future e-education scenario can be provided by implementation of DVB-RCS or some current satellite system and equipment, such as broadband global area network (BGAN) and employing stratospheric communication platforms (SCP). The solutions using BGAN via Inmarsat for small personal or corporate LAN applications and the best solutions for WAN application are SCP or DVB-RCS hub, Interactive VSAT and personal computers with adequate software [9, 22-23].

In Figure 7 is illustrated block diagram of typical rural e-education solution that connects urban and rural areas via DVB-RCS hub, radio frequency (RS) roof antenna and C/Ku/Ka-band GEO spacecraft. On the urban side is ISP connected to router, backbone network via IP lines controlled by the network management system (NMS) and via internet protocol (IP) is linking hub with big dish antenna. The hub is connecting rural e-education schools No 1, No 2, No n and etcetera via GEO C/Ku/Ka-band satellite constellations. The rural schools can be equipped with VSAT routers, antenna and many PC terminals in LAN, WiMAX or WiFi networks. In every school pupils are watching live lecture at their PC monitors or videoconference screens.

Besides, the alternative solutions for e-education and distance learning in rural area can be establishment of mobile e-learning deploying special vehicles or buses by implementing the same DVB-RCS equipment illustrated in the Figure 5. In the similar way, can be used the solutions of VDVoIP in and outdoor units, satellite indoor and handheld telephones, satellite IPPC, IPTV and internet kiosk. However, the problem of the powers supply in the rural schools and remote cities can be solved by installation of some alternative electrical power supply devices, such as electrical generators, solar cells or wind turbines. 


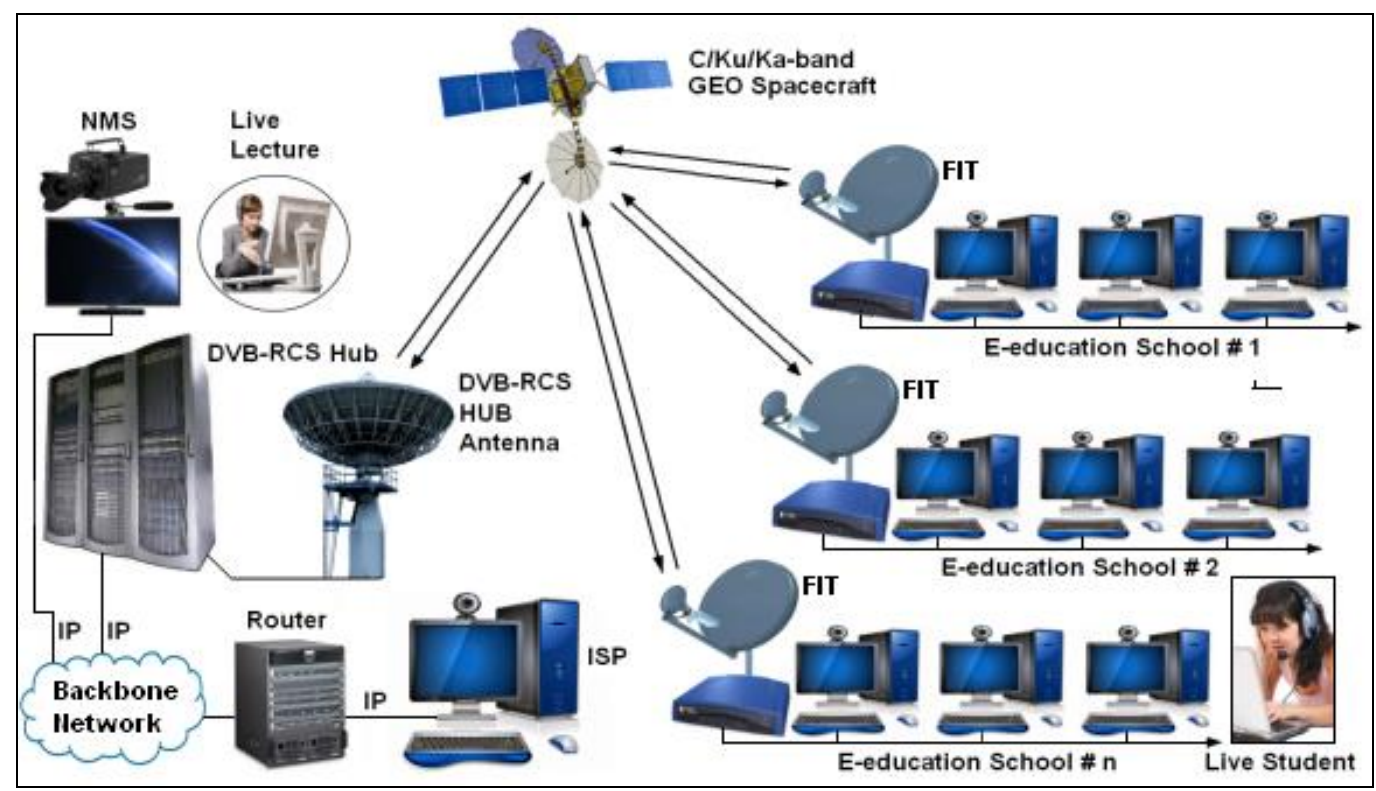

Figure 7. E-education in rural schools via DVB-RCS standards

Each e-education vehicle integrates complete VSAT indoor (IDU) routers and on top of vehicle are installed VSAT outdoor (ODU) reflector antennas at C (4-8 GHz), Ku (12-18 GHz) or Ka-band (27-40 GHz). The VSAT equipment is connecting several PC configurations in LAN inside vehicles or via cables inside schools for interactive videoconference with broadcast e-learning centre (BES) in urban area. Educator is providing live lecture of remote education of any subject for each rural school, portable or mobile e-learning solution. Otherwise, the rural area contains the number of remote schools, which are equipped with VSAT routers, antenna and many PC terminals in LAN, WiMAX or WiFi networks. In every rural school pupils (live students) are watching live lecture at their PC monitors or large videoconference screens. In the same time urban and suburban schools can have the same live lecture directly via Internet connected to the VSAT eeducation network. Therefore, many live lectures located in broadcasting education centre (BEC) can provide lecturing in any subject for the same grade classrooms with live students. At the end of live lecture in BEC for each particular subject classroom teacher is asking pupils for any misunderstandings or additional explanations and in such a way can transfer all questions to the educator in BEC studio for interactive discussion and answers on all questions $[9,10,24-25]$.

\section{CONCLUSION}

The new DVB-S2 is designed to minimize overall system costs for service providers and system operators. This network offers the lowest system costs on the market today for multiple access systems managing VoIP, VDVoIP, IPPC and IPTV multimedia, broadcast and broadband contents. The e-education solution throughout DVB-RCS will improve education and learning facilities in rural and urban areas and will help for better knowledge, information system and service delivery. In addition, DVB-RCS network also will provide distance training facilities for corporate and government requirements. These solutions via DVB-RCS will also help all schools, pupils and teacher to be better managed, controlled, inspected and educated countrywide. In all events, the implementation of DVB-RCS architecture will also improve communication and Internet facilities in rural and remote areas for government and private corporation including emergency, disaster, security, education, health solutions and other e-solutions.

\section{REFERENCES}

[1] Aragón-Zavala A., et al., "High-altitude platforms for wireless communications," John Wiley, Chichester, 2008.

[2] Maral G., "VSAT networks", John Wiley, Chichester, 2003.

[3] Ilcev D. S., "Presentation of mobile DVB-RCS," Manual, DUT, Durban 2008.

[4] Ilcev D. S., "Global mobile satellite communications for maritime, land and aeronautical applications," Springer, Boston, 2005.

[5] Ilcev D. S., "Global mobile satellite communications for maritime, land and aeronautical applications (volume $1 \& 2$ ), Springer, Boston, 2016.

\footnotetext{
Implementation of e-education in Africa via space digital video broadcasting system (Dimov Stojce Ilcev)
} 
[6] Ilcev D. S., "Stratospheric communication platforms (SCP) as an alternative for space program," AEAT Journal, Emerald, Bingley, 2011.

[7] Ilcev D.S., "Communication stratospheric platforms", Journal of the Institute of Telecommunications Professionals, Sunbury on Thames, 2010.

[8] Ilcev D.S., "E-education using DVB-RCS", Journal of International Telecommunication Professionals, Sunbury on Thames, 2012.

[9] Grace D. \& Others, "Broadband communications via high altitude platforms," John Wiley, Chichester, 2011.

[10] Minoli D., "IP Multicast with applications to IPTV and mobile DVB-H," John Wiley, Chichester, 2008.

[11] Evans B.G., "Satellite communication systems," IEE, London, 1991.

[12] Richharia M., "Mobile satellite communications-principes and trends," Addison-Wesley, Harlow, 2001.

[13] Ohmori S. \& others, "Mobile satellite communications," Artech House, Boston, 1998.

[14] Littman M. K., "Building broadband networks," CRC Press, Boca Raton, FL, 2002.

[15] Pollack M., "The key benefits and advantages of DVB-RCS/S2 technologies," Advantech, Montreal, 2006.

[16] Alessi S. \& Trollip S., "Multimedia for learning, methods and development," Allyn \& Bacon, Boston, MA, 2001.

[17] Ciampa M., "Guide to wireless communications," Thomson Course Technology, Boston, MA, 2002.

[18] Dean T., "Guide to telecommunications technology," Thomson Course Technology, Boston, MA, 2002.

[19] NanoTronix, “Two-way VSAT for E-learning," Seoul, [Online], Avalaible: www.nano-tronix.com, 2011.

[20] ViaSat, "DVB-RCS LinkStar System Description", Issue 4, ViaSat, Duluth, 2007.

[21] STM, "SatLink carrierclass," STM DVB-RCS Solutions, Lysaker, 2007.

[22] Advantech, "SatNet DVB-RCS an Outline Proposal," Broadband Solutions, Montreal, 2007.

[23] WebPages: "GEO satellite operators: Intelsat, panamsat, ses-newskies \& Eutelsat," DVB-RCS Solutions, 2018.

[24] WebPages: "GEO Satellite Operators," Eutelsat and Arabsat, 2018.

[25] ViaSat, "DVB-RCS Remotes," [Online], Available: www.viasat.com \& www.hughes.com, 2019.

\section{BIOGRAPHY OF AUTHOR}

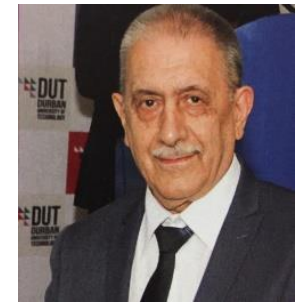

Prof. Dimov Stojce Ilcev is research leader of the Space Science Centre (SSC) for research and postgraduate studies at Durban University of Technology (DUT). He has three BSc degrees in Radio, Nautical Science and Maritime Electronics and Communications. He got MSc and PhD in Mobile Satellite Communications and Navigation as well. Prof. Ilcev also holds the certificates for Radio operator 1st class (Morse), for GMDSS 1st class Radio Electronic Operator and Maintainer and for Master Mariner without Limitations. He is author of several books in mobile Radio and Satellite CNS, DVB-RCS, Satellite Asset Tracking (SAT), Stratospheric Platform Systems (SCP) for maritime, land (road and railways) and aeronautical applications. 